\title{
An Overview of Text Summarization
}

\author{
Laxmi B. Rananavare \\ Research Scholar \\ S V U College of Engg \\ Tirupathi, Andhra Pradesh, India-517502
}

\author{
P. Venkata Subba Reddy \\ Professor \\ S V U College of Engg \\ Tirupathi, Andhra Pradesh, India-517502
}

\begin{abstract}
The amount of e-information available has increased greatly over the past few decades. As the vast amount of information is available for every theme on Internet, shortening the information in the form of summary would immensely benefit readers. Hence, the natural language processing research community is developing new methods for summarizing the text mechanically. Automatic text summarization system produces a summary, i.e. short length text that includes all the significant information for the article. This paper presents a comprehensive survey of contemporary text summarization of extractive and abstractive approaches.
\end{abstract}

\section{Keywords}

Text Summarization

\section{INTRODUCTION}

The goal of automatic summarization is to take e-information, extract the important content from it and present the content to the user in a condensed form and in a manner amenable to the user's or application's need [75]. Because of world wide web, mobile communication we have access to large amount of information. We have more information with less time or ability to go through it. So there is need for automatic summarization in such situation. The amount of information on the web is in diverse forms such as text, video, images. it is very difficult for users to come upon important information of his/her interest. Automatic summarization field exists since 1950. Researchers have been trying to devise techniques for generating summaries so that machine generated summary would replace the human-made summary. There are two approaches for generating summary: extractive and abstractive. An extractive method builds summary using selected and juxtaposed sentences from the original text. An abstractive method regenerates the gist of a text in the form of new sentences. Abstractive methods are highly complecated and difficult as they need understanding of the document. The generation of abstracts is an intellectual effort and it requires familiarity with the subject. Researchers are focusing more on a simpler approach based on extractive summaries and trying to achieve more coherent and cohesive summaries. Summaries can be viewed in terms indicative and informative abstracts. An indicative abstract gives comprehensive information that helps the user to decide whether to read the document or not. An informative abstract covers all the important information source. Depending on the type user the summary can be catagorized as User-focused summaries (or Topic focused or query focused) and generic summaries. User-focused summaries are tailored to requirements of a particular user or group users. Generic summaries are aimed at particular reader community. These summaries may be indicative or informative. Depending on the number of documents the summarization can be classified as single document or multiple document summarization. Automatic summarization shortens a source document into meaningful content without altering information. Thus it helps the user to grasp the main concept quickly without the need to examine the whole document, thereby saving time and efforts. Text summarization process works in three steps [35] as follows,

-Analysis step analyzes source text and selects attributes.

- Transformation step transforms the result of the analysis.

- Synthesis step produces the summary.

This paper presents extractive, abstractive text summarization techniques and different evaluation methods for text summarization. The paper is organized as follows: Section II describes extractive summarization techniques. Section III describes abstractive summarization techniques. Section IV Evaluation methods. Section V concludes the paper.

\section{EXTRACTIVE TEXT SUMMARIZATION METHOD}

These are also called shallow approaches wherein they do not venture beyond a syntactic level of representation. For example, words may be analyzed to a semantic level but sentences will be analyzed at most to asyntactic level. The advantage of this method is robustness. The challenge in extractive summarization is to decide which sentences from the input document are important and should be included in the summary. Extractive methods [84] has three steps:

- Create an intermediate representation of the original text;

- Sentence scoring;

- Select high scores sentences to the summary.

First step creates a representation of the document. Usually, it divides the text into paragraphs, sentences, and tokens. Sometimes some preprocessing, such as stop word removal is performed. The second step tries to determine which sentences are important to the document or to which extent it combines information about different topics, by sentence scoring. It first, assigns a score to each sentence based on a feature such as Word scoring, Word Frequency, Term Frequency-Inverse Document Frequency(TF/IDF ), Lexical Similarity, and Sentence Length then ranks sentences according to 
their score. The score should be a measure of how significant a sentence is to the understanding of the text as a whole. The last step combines the score provided by the previous steps and generates a summary. The following definitions presents the main methods in each of the aforementioned approaches,

-Word scoring:The initial methods in sentence scoring were based on words. Each word receives a score and the weight of each sentence is the sum of all scores of its constituent words. The approaches in the literature are outlined here.

-Word frequency: As the name of the method suggests, the more frequently a words occurs in the text, the higher its score. In other words, sentences containing the most frequent words in a document stand a higher chance of being selected for the final summary. The assumption is that the higher the frequency of a word in the text, the more likely that it indicates the subject of the text.

- TF/IDF: This algorithm performs a comparison between the term frequency (TF) in a document (in this case each sentence is treated as a document) and the document frequency (IDF), which means the number of times that the word occurs along all documents.

- Lexical similarity: It is based on the assumption that important sentences are identified by strong chains. In other words, it relates sentences that employ words with the same meaning (synonyms) or other semantic relation.

- Sentence length: This feature is employed to penalize sentences that are too short. The method uses length as number of words in sentence. In addition, penalizes sentences that are shorter than a certain length.

\subsection{Term Frequency - Inverse Document Frequency Method (TF*IDF)}

The TF*IDF is a numerical statistic that is intended to reflect how important a word is to a document in a collection or corpus. Then sentences are scored according to product and sentence having high score are included in the summary. The problem with this method is that longer sentences get higher scores than shorter sentences. This is a consequence of having more words in longer sentences thereby summary length increase. At IBM, Luhn[69] worked on technical documents. In this work he removed stop word in the document and frequency of content words was considered in generating summary. This method looks for concentration of salient (content) words in the document. He calculated the score of sentences as follows,

$$
\text { score }=\frac{\text { Number of significant words in the sentence }}{\text { number of words }}
$$

Edmundson[18] presented a weight based method wherein the basic unit of extraction is the sentence. The method computes the weight of each sentence based on certain features like location, title, cue words, stigma words and keywords. A sentence is given weight based on its location in the document. This feature is dependent on the type of the document. For example, in technical documents, sentences in the conclusion section are ranked high, while in news articles; first few sentences are ranked higher. Sentences containing title words are considered to have a higher score. Title words are those that are present in the title of the document, headings and subheadings are significant words are given higher scores. Cue words are those words containing cue words/phrases like conclusion, concisely etc. They add a positive score to the word. Stigma words are those words that add a negative score to the word. Words like slowly etc. come under this category. Keywords are the words that tend to be more redundant and talk about the main content in the given text. Score of a sentence is then computed as the sum of the scores of its constituent words. Jayashree $\mathrm{R}$ et al.[47] developed an algorithm that extracts key words from Kannada text documents, for which they combine GSS ( Galavotti, Sebastiani, Simi) coefficients and IDF(Inverse Document Frequency) methods along with TF(Term Frequency) for extracting key words and later uses these for summarization. The principal objective of this work is to assign a weight to each word in a sentence. The weight of a sentence is the sum of weights of all words, based on the scoring of sentences. They choose top ' $m$ ' sentences for generating a summary of length m. Mikael Kageback et al. [49] proposed the use of continuous vector representations for semantically aware representations of sentences as a basis for measuring similarity. They investigated the effects of using phrase embedding for summarization. Two implementations of word vectors and two different approaches for composition were evaluated. All investigated combinations improved the original Lin-Bilmes approach (using tf-idf representations of sentences). Rafael Ferreira et al. [23] used three different corpora. One would obtain a coincidence of four methods as being the best ones: Word Frequency, TF/IDF, Lexical Similarity, and Sentence Length. The strategy Text- Rank Score was also chosen by as providing good results for two of the three data sets tested. Chin-Yew Lin et al. [60] represented a procedure to automatically acquire topic signatures and evaluates the effectiveness of applying topic signatures to extract topic relevant sentences. The topic signature method outperforms the baseline and the $\mathrm{tf} * \mathrm{idf}$ methods for all test topics. Topic signatures can not only recognize related terms (topic identification), but group related terms together under one target concept (topic interpretation). Topic signatures have also been viewed as an inverse process of query expansion. A Progressive summary benefits a user to monitor changes in evolving news topics over a period. Detecting innovative information is the essential part of advanced summarization that differentiates it from normal multi- document summarization. Praveen Bysani [10]explores the possibility of detecting novelty at various stages of summarization. New scoring features, Re-ranking criteria, and filtering strategies are proposed to identify relevant novel information. Jiaming Zhan et al. [120] propose an approach to automatically summarize multiple customer reviews based on their internal topic structure. This method accounts for topical overlap within real-world reviews by extracting topics across reviews, instead of dividing reviews into several non-overlapping clusters. The experimental study and its evaluation results have demonstrated that the proposed approach can achieve better summarization performance and users satisfaction when compared to the approaches of opinion mining and clustering-summarization. Atefeh Farzindar et al. [22] describe a method for the summarization of legal documents helping a legal expert determine the key ideas of judgement. The approach relies on the exploration of the documents architecture and its thematic structures to build a table style summary for improving coherency and readability of the text. The method extracts relevant units in the source judgment by identifying the discourse structures and determining the semantic roles of thematic segments in the document. The thematic structures in a tabular form divide the summary presentation: DECISION DATA, INTRODUCTION, CONTEXT, JURIDICAL ANALYSIS and CONCLUSION. The generation of the summary is done in four steps: thematic segmentation to detect the document structures, filtering to eliminate unimportant quotations and noises, selection of the candidate units and production of table style summary. Orkut Buyukkokten et al. [9] discuss a new approach to summarize and browse Web pages on small devices. Each Web page is broken into text units that can each be hidden, partially displayed, made fully visible, or summarized. One method 
extracts significant keywords from the text units, another attempts to find each text units most significant sentence to act as a summary for the unit. The model uses information retrieval techniques, which adapts to the World-Wide Web context. Rafeeq Al-Hashemi [1] generated summarization in four stages. The preprocess stages convert the unstructured text into structured. In the first stage, the system removes the stop words, parse the text and assigning the POS (tag) for each word in the text and store the result in a table. The second stage extracts the relevant key phrases in the text and rank the candidate words. The system uses the extracted keywords/keyphrases to select the important sentence. Each sentence ranked depending on many features such as the existence of the keywords/keyphrase in it, the relation between the sentence and the title by using a similarity measurement and other many features. The Third stage of the proposed system is to extract the sentences with the highest rank. The Fourth filtering stage reduces the number of the candidate sentences, in summary, to produce a qualitative summary using KFIDF measurement. The work presented here depends on the keyphrases extracted by the system and many other features extracted from the document to get the text summary as a result. This approach provides an advantage of finding the most related sentences to be added to the summary text. The system gave good results in comparison to manual summarization extraction. The system can provide the most compressed summary with high quality. The main applications of this work are Web search Engines, text compression, and word processor. Inderjeet Mani et al. [72] explored different cohesion methods for computing salience, and compared these methods against a coherence-based method regarding accuracy with respect to human judgements of salience. Despite the lack of links based on proper name anaphora and synonymy in these texts, the results of the spreading-based cohesion method, while less accurate overall than the coherence method, and are encouraging. Further, a method for discovering discourse-related text structure based on local cohesion was introduced. It also compares such trees with coherence-based trees. Rada Mihalcea et al. [12] tackled the problem of book summarization. they introduced a new summarization benchmark, specifically targeting the evaluation of systems for book summarization. They showed that systems developed for the summarization of short documents do not farely well when applied to very long documents such as books, and instead, a better performance can be achieved with a system that accounts for the length of the documents.

\subsection{Cluster- Based Methods}

Documents are composed in such a manner that they address different ideas in separate sections. It is natural to think that summaries should address different themes separated into sections of the document. In case that the document for which summary is being delivered is of entirely different subjects then summarizer assimilates this aspect through clustering. The document is represented using TF-IDF of scores of words. High- frequency term represents the theme of a cluster. Summary sentence is selected based on relationship of sentence to the theme of the cluster. The cluster- based method generates a summary of high relevance, to the given query or document topic. Chris Kedzie et al. [51] have presented an update summarization system for the disaster domain, and demonstrated improved system performance by integrating sentence salience with clustering. They use novel, disasterspecific features for salience prediction, including geo-locations and language models representing the language of disaster. Vahed Qazvinian et al. [92] propose a model of summarizing a single article, which can be further used to summarize an entire topic.
This work uses the citation summaries to understand the main contributions of articles and proposes a clustering approach where communities in the citation summarys lexical network are formed and sentences are extracted from separate clusters.Ramiz $\mathrm{M}$ et al. [2]presented the approach to automatic document summarization based on clustering and extraction of sentences. The approach consists of two steps. First sentences are clustered, and then on each cluster representative sentences are defined. A discrete differential evolution algorithm to optimize the objective functions is developed. This paper also demonstrated that the summarization result depends on the similarity measure. Results of the experiment have shown that proposed by us NGD-based dissimilarity measure outperforms the Euclidean distance. Hiroya Takamura et al. [107] proposed a novel text summarization model based on the budgeted median problem. The proposed model covers the entire document cluster through sentence assignment, since in this model every sentence is represented by one of the selected sentences as much as possible. An advantage of the method is that it can naturally incorporate asymmetric relations between sentences.Dragomir Radev et al. [94] use a form of multi-document summarization called Centroid-based summarization. A cluster of documents with a common topic is used to produce a cluster centroid, consisting of words which are central to all of the documents in the cluster. The cluster centroid is then used to rank sentences based on their relevance to the topic of the cluster. Dragomir R. Radev et al. [96] present a multi-document summarizer, called MEAD, which generates summaries using cluster centroids produced by a topic detection and tracking system. It also describes two new techniques, based on sentence utility and subsumption. It summarizes clusters of news articles automatically grouped by a topic detection system. P. Arun Kumar et al. [54] proposed an improved text summarization approach by combining popularity and weight measures. Note that the popularity of a sentence is determined by the number of similar sentences in the text which is a cluster. Certain features like the position of the sentence, the presence of cue words, etc. measure the weight of a sentence. The methods, when applied independently, fail to select all the salient sentences. By combining the above two methods, it is possible to improve the performance. N K Nagwani [80] designed a technique using semantic similarity based clustering and topic modeling using Latent Dirichlet Allocation (LDA) for summarizing the large text collection over MapReduce framework. The summarization task is performed in four stages and provides a modular implementation of multiple documents summarization. The presented technique is evaluated in terms of scalability and various text summarization parameters namely, compression ratio, retention ratio, ROUGE and Pyramid score are also measured. MapReduce provides a faster implementation of summarizing large text collections and is a powerful tool in Big Text Data analysis.

\subsection{Text Summarization with Neural Network}

A Neural Network is a processing system modeled on the human brain that tries to reenact its learning process. The neural network is an interconnected assembly of artificial neurons that uses a numerical model of computation for data processing. In the case of text summarization, the strategy includes preparing the neural systems to capture the sort of sentences that ought to be incorporated into the summary. Neural Network is trained with sentences in test paragraph where each sentence is checked as to be included in summary or not. Training is conducted by the need of the user. Neural network accurately classifies summary sentences but faces the problem of excessive training time. Khosrow Kaikhah [50] presented a novel technique for summarizing news articles using neural net- 
works. The neural network is modified to generalize and combine the relevant features apparent in summary sentences. Finally, the modified neural network is used as a filter to summarize news articles. The performance of the text summarization process depends predominantly on the style of the human reader. The selection of features, as well as the selection of summary sentences by the human reader from the training paragraphs, play a major role in the performance of the network. The network is trained according to the style of the human reader and to which sentences the human reader deems to be important in a paragraph. Individual readers can train the neural network according to their own reading styles. The selected features can be modified to reflect the readers needs and requirements. Baotian Hu Qingcai Chen Fangze Zhu [43] introduced a large corpus of Chinese short text summarization dataset constructed from the Chinese microblogging and performed Recurrent Neural Network-based methods with promising results. A recurrent neural network (RNN) is a class of artificial neural network creates an internal state of the network which allows it to exhibit dynamic temporal behavior. The rare word problem is also significant for the generation of the summaries, especially when the input is wordbased instead of character-based. It is also a hot topic in the neural generative models such as neural translation machine (NTM) (Luong et al., 2014) set by using naturally annotated web resources. Jayashree $\mathrm{R}$ et al. [46] discuss a machine learning approach that uses artificial neural networks to produce summaries of arbitrary length text documents. A feed forward neural network, also called as back propagation network, is trained on a corpus of text documents. The corpus is custom built for this purpose using Kannada web portals. Oriol Vinyals et al.[112] Conversational modeling is an important task in natural language understanding and machine intelligence. The paper uses the recently proposed sequence to sequence framework. The model converses by predicting the next sentence given the previous sentence or sentences in a conversation. The strength of the model is that it can be trained end-to-end and thus requires much fewer hand-crafted rules. The modest results show that it can generate simple and basic conversations, and extract knowledge from a noisy but open-domain dataset. A purely data- driven approach without any rules can produce rather proper answers to many types of questions. However, the model may require substantial modifications to be able to deliver realistic conversations. Amongst the many limitations, the lack of a coherent personality makes it difficult for this approach to pass the Turing test (Turing, 1950).

\subsection{Text Summarization with Fuzzy Logic}

Fuzzy Logic is a way of reasoning that resembles with the human reasoning which based on degrees of truth rather than the usual true or false ( 1 or 0 ) Boolean logic. The fuzzy system is designed with fuzzy rules and membership function which highly affect the performance. A value from zero to one is obtained for each sentence in output based on feature contained in sentence and rules defined in a knowledge base. Meaningful sentences are extracted using IFTHEN rules based on feature criteria. Sentences are ranked in order according to score. In summary, sentences having high score are extracted. Fuzzy logic systems are simple and flexible can take imprecise, distorted, noisy input information. Ladda Suanmali et al. [106] present a fuzzy logic aided sentence extractive summarizer that can be as informative as the full text of a document with better information coverage. A prototype has also been constructed to evaluate this automatic text summarization scheme using as in- put some news articles collection provided by DUC2002 $2^{1}$ It extracts the important features for each sentence of the document represented as the vector of features consisting of the following elements: title feature, sentence length, term weight, sentence position, sentence to sentence similarity, proper noun, thematic word and numerical data. Certainly, the experimental result is based on fuzzy logic could improve the quality of summary results that based on the general statistic method. F. KYOOMARSI et al. [53] present a new approach for creating summaries using fuzzy inference system. The analysis of the parameters which are important in summarization was done by a number of fuzzy-logic-based analyzers. This text summarization system consists of 1) text pre-processor which extracts different information needed for fuzzy analysis from the text using the word-net database and 2) analyzers which contain fuzzy-logic-based inference systems to compute the weighted score of each sentence in the text. The scores of relevance have been ranked. Starting with the highest score, the analyzer includes in the summary the sentences for which the relevance score is greater than the threshold value set. Evaluation using ROUGE indicates the advantage of this approach in comparison to referencing human summarizations. The weakness of the proposed fuzzy summarizer is that the process of designing fuzzy rules, which have to cover all the relationships among the parameters, is quite time- consuming. $\mathrm{Po} \mathrm{Hu}$ et al. [44] propose a novel unsupervised approach by making use of enhanced social context to aid personalized summary generation. In the proposed method, document expansion, user expansion, and implicit induction of the intended user's interest aspects are achieved simultaneously by adopting a fuzzy tripartite clustering algorithm. Moreover, both the informativeness of sentences and the users interest aspects are incorporated in a unified ranking process.

\subsection{Graph- based Method}

In this method, the sentences of the document are considered as a vertices of the graph. these sentences are connected with an edge if there exist common semantic relation and based on this relation connecting edge is given weight. A graph-based ranking algorithm is used to decide the importance of a vertex within a graph. Vertexes with high cardinality are considered as important sentences and included in the summary. The graph-based method requires neither a profound linguistic knowledge, nor domain knowledge for summarization. Directed graph maintains a flow of text while an edge in undirected graph captures relation using co-occurrence of term. Tree is acyclic graph. Masaaki Nishino et al. [87 proposed a dynamic programming (DP) algorithm for tree trimming problems with time complexity of $\mathrm{O}(\mathrm{NL} \log \mathrm{N})$, where $\mathrm{N}$ represents the number of tree nodes and $\mathrm{L}$ is the length limit. The algorithm exploits the zero-suppressed binary decision diagram (ZDD), a data structure that accounts for a family of sets as a directed acyclic graph. ZDD permits the application of DP to obtain exact solutions and is faster than ILP solvers. Experiments show that the proposal allows three different tree trimming problems to be solved in the same way. Research of Wauter Bosma [7] presents a framework for graph-based summarization to model relations in text, so that the passages can be viewed in a broader context. The result is a summarization system which is more in line with discourse theory but still fully automatic. The aim of this paper is to bring automatic summarization practice in line with insights from discourse theory. To this end, it provides a framework for automatic summarization which is founded on graph theory. The system sig-

${ }^{1}$ Document Understanding Conferences 
nificantly outperforms a competitive baseline (and participant systems) on the DUC 2005 evaluation set. The content selection algorithm is entirely based on relations between text passages. The evaluated system is just one implementation of this framework. Anand Gupta et al. [32] introduced a novel method for single document text summarization. The model poses the text summarization task as an optimization problem, and attempts to solve it using Weighted Minimum Vertex Cover (WMVC), a graph-based algorithm. Textual entailment, an established indicator of semantic relationships between text units, is used to measure sentence connectivity and construct the graph on which WMVC operates.Rada Mihalcea [76] presents an innovative unsupervised method for automatic sentence extraction using a graph- based ranking algorithm called TextRank which identifies connections between various entities in a text, and implements the concept of recommendation. A text unit recommends other related text units, and the strength of the recommendation is recursively computed based on the importance of the units making the recommendation. In the process of identifying significant sentences in a text, a sentence recommends another sentence that addresses similar concepts as being useful for the overall understanding of the text. Sentences that are highly recommended by other sentences are likely to be more informative for the given text, and will be therefore given a higher score. An important aspect of TextRank is that it does not require deep linguistic knowledge, nor domain or language specific annotated corpora, which makes it highly portable to other domains, genres, or languages. Hongyuan Zha[119] A novel method for simultaneous keyphrase extraction and generic text summarization is proposed by modeling text documents as weighted undirected and weighted bipartite graphs. Spectral graph clustering algorithms are used for partitioning sentences of the documents into topical groups with sentence link priors being exploited to enhance clustering quality. Within each topical group, saliency scores for key phrases and sentences are generated based on a mutual reinforcement principle. The key phrases and sentences are then ranked according to their saliency scores and selected for inclusion in the top keyphrase list and summaries of the document. The idea of building a hierarchy of summaries for documents capturing different levels of granularity is also briefly discussed. Gunes Erkan, Dragomir R. Radev[20] presented a new approach, LexRank, for computing sentence importance based on the concept of eigenvector centrality in a graph representation of sentences is used. In this model, a connectivity matrix based on intra-sentence cosine similarity is used as the adjacency matrix of the graph representation of sentences. The paper discusses several methods to compute centrality using the similarity graph. The results show that degree-based methods (including LexRank) outperform both centroid-based methods and other systems participating in DUC in most of the cases. Furthermore, the LexRank with threshold method outperforms the other degree-based techniques including continuous LexRank. This approach is quite insensitive to the noise in the data that may result from an imperfect topical clustering of documents.Kavita Ganesan et al. [25] presented a novel graph-based summarization framework (Opinosis) that generates concise abstractive summaries of highly redundant opinions that uses textual graphs to generate abstractive summaries of highly redundant opinions. Evaluation results on summarizing user reviews show that Opinosis summaries have better agreement with human summaries compared to the baseline extractive method. The summaries are readable, reasonably wellformed and are informative enough to convey the major opinions. This paper explains the task of multi-tweet summarization, which selects a given number of representative tweets so as to keep important information while dropping noise and redundancy. One main motivation of this task is to provide a tool to help people efficiently access a large number of tweets, which are short and prone to noise. This is important considering that tweets are one increasing popular repository of fresh information. Inderjeet Mani et al. [71] describe a new method for summarizing similarities and differences in a pair of related documents using a graph representation of text. Concepts denoted by words, phrases, and proper names in the document are represented positionally as nodes in the graph along with edges corresponding to semantic relations between items. Given a perspective in terms of which the pair of documents is to be summarized, the algorithm first uses a spreading activation technique to discover, in each document, nodes semantically related to the topic. The activated graphs of each document are then matched to yield a graph corresponding to similarities and differences between the pair, which is rendered in natural language. An evaluation of these techniques has been carried out. The summarization exploits the results of recent progress in information extraction to represent salient units of text and their relationships. By exploiting relations between units and the perspective from which the comparison is desired, the summarizer can pinpoint similarities and differences. This approach is highly domain-independent, even though it has illustrated its power mainly for news articles. Currently, the synthesis component is rudimentary, relying on sentence extraction to exemplify similarities and differences.Rada Mihalcea et al. [77] described a method for language independent extractive summarization that relies on iterative graph-based ranking algorithms. Intuitively, iterative graph-based ranking algorithms work well on the task of extractive summarization because they do not only rely on the local context of a text unit (vertex), but they rather take into account information recursively drawn from the entire text (graph). Through the graphs it builds on texts, a graph-based ranking algorithm identifies connections between various entities in a text, and implements the concept of recommendation. A text unit recommends other related text units, and the strength of the recommendation is recursively computed based on the importance of the units making the recommendation. In the process of identifying relevant sentences in a text, a sentence recommends another sentence that addresses similar concepts as being useful for the overall understanding of the text. Sentences that are highly recommended by other sentences are likely to be more informative, and will be, therefore, given a higher score. Hongyan Jing [48] present a novel sentence reduction system for automatically removing extraneous phrases such as prepositional phrases, clauses, to-infinitives, or gerunds, and multiple phrases can be removed form a single sentence that is extracted from a document for summarization purpose. The system uses multiple sources of knowledge to decide which phrases in an extracted sentence can be removed, including syntactic knowledge, context information, and statistics computed from a corpus which consists of examples written by human professionals. The reduction can significantly improve the conciseness of automatic summaries. The system makes intelligent reduction decisions based on multiple sources of knowledge, including syntactic knowledge, context, and probabilities computed from corpus analysis. LIU X et al. [67] explain the task of multi-tweet summarization, which selects a given number of representative tweets so as to keep important information while dropping noise and redundancy. One main motivation of this task is to provide a tool to help people efficiently access a large number of tweets, which are short and prone to noise. This is important considering that tweets are one increasing popular repository of fresh information. 


\subsection{Latent Semantic Analysis Method}

LSA is algebraic statistical method that extracts meaning and resemblance of a sentence by the information about words in a particular environment. It keeps information about which words are used in sentence and reserve information of common word amongst sentences, the more common word between sentences the more it relevant. LSA extracts the source text and converts into term- sentence matrix and process it through Singular Value Decomposition (SVD) for finding semantically similar words and sentences. SVD models relationships among words and sentences. The key point of LSA is it avoids the problem of synonyms. It uses only information in the input text. It does not use the information of word order. Syntactic relations is the major limitation of this method. Josef Steinberger et al. [105] The core of the measure is covered by Latent Semantic Analysis (LSA) which can capture the main topics of a document. The summarization systems are ranked according to the similarity of the main topics of their summaries and their reference documents. Results show a high correlation between human rankings and the LSA-based evaluation measure. The measure is designed to compare a summary with its full text. It can compare a summary with a human written abstract as well; however, in this case using a standard ROUGE measure gives more precise results. Nevertheless, if abstracts are not available for a given corpus, using the LSA-based measure is an appropriate choice.Leonhard Hennig [39] introduced an approach to query-focused multi-document summarization based on the probabilistic latent semantic analysis. After training a PLSA model on the term-sentence matrix of document clusters from recent summarization tasks, it represents each sentence as a distribution over latent topics. Using this representation, they combine query-focused and thematic sentence features into an overall sentence score. Sentences are ranked and selected for the summary according to this score, choosing a greedy approach for sentence selection and penalizing redundancy with a maximum marginal relevance method. Yihong Gong et al. [30] presented two text summarization methods that create generic text summaries by ranking and extracting sentences from the original documents. The first method uses standard IR methods to rank sentence relevance, while the second method uses the latent semantic analysis tech unique to identify semantically important sentences, for summary creations. Both methods strive to select sentences that are highly ranked and different from each other. This is an attempt to create a summary with a wider coverage of the document's content and a less redundancy. Yeh et al. [116] proposed a trainable summarizer, which takes into account several features, including position, positive keyword, negative keyword, centrality, and the resemblance to the title, to generate summaries. Two new ideas are exploited: (1) sentence positions are ranked to emphasize the significances of different sentence positions, and (2) the score function is trained by the genetic algorithm (GA) to obtain a suitable combination of feature weights. The second uses latent semantic analysis (LSA) to derive the semantic matrix of a document or a corpus and uses semantic sentence representation to construct a semantic text relationship map.

\subsection{Machine Learning approach}

In this method, the training data set is used for reference to generate a summary. Summarization process is modeled as a classification problem. Sentences are classified as summary sentences and non-summary sentences based on the features that they possess. Text summarization algorithms based on machine learning approach such as Naive-Bayes, Decision Trees, Hidden Markov
Model, Log-linear Models, etc. are described by Das et al. [16]. Baxendale[6] proposed machine learning techniques for reducing technical documents to their essential discriminating indices. He used positional method. Usually the first and last paragraphs of document contain topic sentences. Human scanning patterns in selecting topic sentences and phrases composed of nouns and modifiers were simulated by computer program. The amount of condensation resulting from each method and the relative uniformity in indices are examined.Julian Kupiec et al.[55]developed a trainable summarization program which uses statistical framework. They used Nave Bayes classifier and assumes statistical independence performance. For summaries that are $25 \%$ of the size of the average test document, it selects $84 \%$ of the sentences chosen by professionals. This paper focuses on analysis of written text. Thu et al. [110] proposed Bayesian Network that finds important sentence using probability difference. Reduced sentence is generated with the weightiest path from source node to following nodes in node network. Computational cost is reduced using dynamic programming. Research of Sarkar et al. [103] approached the problem of automatically generating summary from medical article as a supervised learning task. This work treats a document as a set of sentences, which the learning algorithm must learn to classify as positive or negative examples of sentences based on summary worthiness of the sentences. Then they apply the machine learning algorithm called bagging to this learning task, where a decision tree has been chosen as the base learner. They also compare the proposed approach to some existing summarization approaches.Geraldd Ejong[17] developed a knowledge based program(slot filling approach), called FRUMP for Fast Reading Understanding and Memory Program, and employs this approach to parsing. It is based on 50 sketchy scripts. The input is matched to script based on manually selected keywords. FRUMP skims articles rather than reading them for detail. The program works on the relatively unconstrained domain of news articles. It routinely understands stories it has never before seen. The program's success is largely due to its radically different approach to parsing. It is difficult to port this program to other domains. Also there are missing scripts to many inputs. Yu et al. [118] proposed machine learning approach that uses Hidden Markov Models (HMM), Conditional Random Field (CRF), Gaussian Mixture Models (GMM) and Mathematical Methods of Statistics (MMS) method for cross-language summarization text. The model is trained with the feature vector. Character based tagging is used with all proposed methods to generate a summary. Annie Louis [68] introduced a Bayesian summarization method that strongly aligns with intuitions about how people use existing knowledge to identify important events or content in new observations. This method utilizes Bayesian surprise which provides an intuitive way to identify surprising information from a summarization input with respect to a small background corpus. The system uses smaller amounts of background information and as new data arrives, be able to incorporate the evidence. Ivan Titov et al. [111] presented a joint model of text and aspect ratings for extracting text to be displayed in sentiment summaries. They proposed a statistical model which can discover corresponding topics in text and extract textual evidence from reviews supporting each of these aspect ratings. More formally, they consider the log-linear distribution. The model uses aspect ratings to discover the corresponding topics and can thus extract fragments of text discussing these aspects without the need of annotated data. The work demonstrates that the model discovers corresponding coherent topics and achieves accuracy in sentence labeling comparable to a standard supervised model. Online reviews are often accompanied with numerical ratings provided by users for a set of service or product 
aspects. Markus Zopf et al. [122] proposed CPSum (content-word pairs summarizer), a summarizer that learns the importance of information objects from a background source. CPSum, a text summarization system that learns the importance of entities from an independent background corpus of document-summary pairs. CPSum is able to cope with summarization scenarios where neither centrality nor structural features that help to detect important information. The hypothesis is tested on a multi-document corpus where they removed centrality and structural features. Vahed Qazvinian et al. [93] present an approach to summarize single scientific papers, by extracting its contributions from the set of citation sentences written in other papers. The methodology is based on extracting significant key phrases from the set of citation sentences picks sentences to cover nuggets (represented by keyphrases) of single paper summarization, and how it out-performs other multi-document summarization methods. In this work, they use pointwise KLdivergence to extract statistically significant Ngrams and use them to represent nuggets. They apply a new set function for the task of summarizing scientific articles Vishal Gupta et al. [33] discussed the condition based named entity recognition approach for Punjabi text summarization system. Most of the lexical resources used in NER such as prefix list, Suffix list, Middle name list, Last name list and Punjabi proper name list had to be developed from scratch as no work had been done in that direction. In condition- based approach, five rules have been implemented like prefix rule, Suffix rule, Middle name rule, last name rule and proper name rule which uses HMM. Jade Goldstein et al. [29] presented the analysis of newsarticle summaries generated by sentence selection. Sentences are ranked for potential inclusion in summary, using a weighted combination of statistical and linguistic features. The statistical features were adapted from standard IR methods. The potential linguistic ones were derived from an analysis of news-wire summaries. To evaluate these features, they use a normalized version of precisionrecall curves, with a baseline of random sentence selection, as well as analyze the properties of such a baseline. Yulia Ledeneva et al. [57] this work shows experimentally that words that are parts of bigrams that repeat more than once in the text are good terms to describe the texts contents, and so are also so-called maximal frequent sentences. It also shows that the frequency of the term as term weight gives good results. KUO-EN CHANG et al. [13] Although graphic strategies, such as graphic organizers and knowledge maps, have proved helpful for text learning, certain important application issues such as surface processing and cognitive overload have yet to be resolved. The authors tested the learning effects of a conceptmapping strategy. They designed three concept-mapping approachesmap correction, scaffold fading, and map generation to determine their effects on learners text comprehension and summarization abilities. The experimental results from 126 fifth graders showed that the map-correction method enhanced text comprehension and summarization abilities and that the scaffold-fading method facilitated summarization ability. SHIXIA LIU et al. [65] This paper introduces an enhanced, LDA (Latent Dirichlet Allocation) based topic analysis technique that automatically derives a set of topics to summarize a collection of documents and their content evolution over time. This work offers three unique contributions. First, it presents an enhanced topic modeling technique to provide users with a time-sensitive and more meaningful text summary. Second, it develops an effective visual metaphor to transform abstract and often complex text summarization results into a comprehensible visual representation. Third, it offers users flexible visual interaction tools as alternatives to compensate for the deficiencies of current text summarization techniques. Asli Celikyilmaz et al. Tur [11] this paper formulates an extractive summarization as a two-step learn- ing problem building a generative model for pattern discovery and a regression model for inference. It calculates scores for sentences in document clusters based on their latent characteristics using a hierarchical topic model. Then, using these scores, it trains a regression model based on the lexical and structural characteristics of the sentences, and use the model to score sentences of new documents to form a summary. Jun Ping $\mathrm{N} g$ et al. [86] show that by making use of information common to document sets belonging to a common category, one can improve the quality of automatically extracted content in multi-document summaries. The CSI has two features: category relevance score (CRS), an intra-category measure; and category KL-divergence score (CKLD), an inter-category measure.Aria Haghighi et al. [34] present an exploration of generative probabilistic models for multi-document summarization. Beginning with a simple word frequency based model (Nenkova and Vanderwende, 2005), the model constructs a sequence of models each injecting more structure into the representation of document set content and exhibiting ROUGE gains along the way. This paper presents an exploration of content models for multi-document summarization and demonstrates that the use of structured topic models could benefit summarization quality as measured by automatic and manual metrics. Ani Nenkova et al. [83] addressed the question of what makes the writing of a summary for a multi-document input difficult. Summary length is a significant factor, with all summarizers (people, machines, and baselines) performing better at longer summary lengths.they defined a number of features aimed at capturing input cohesiveness, ranging from simple features such as input length and size to more sophisticated measures such as input set entropy, KL divergence from a background corpus and topic signature terms based on the log-likelihood ratio. Experiments with a logistic regression classifier based on the features further confirms that input cohesiveness is predictive of the difficulty it will pose to automatic summarizers. It is important to develop strategies that can better handle non-cohesive inputs, reducing fluctuations in system performance. Tadashi Nomoto et al. [88] proposed and empirically motivated an integration of supervised learning with unsupervised learning to deal with human biases in summarization. The model explores the use of probabilistic decision tree within the clustering framework to account for the variation as well as regularity in human created summaries. The corpus of humanly created extracts is created from a newspaper corpus and used as a test set. The paper is focused on the use of decision tree as a plug-in learner. Massih-Reza Amini et al. [3] proposed new semi-supervised algorithms for training classification models for text summarization. The work analyzes the performances on two data sets - the Reuters newswire corpus and the Computation and Language collection of TIPSTER SUMMAC. Prakash et al. [91] proposed reinforcement learning where term- sentence matrix for each term in the sentence is calculated using TF*IDF. For scoring the sentences, sentence signature matrix is used. Sentences are selected by calculating cosine angle of matrix reinforcement learning to generate a summary. Wesley T. Chuang et al. [15] adopted a method wherein sentences are broken into segments by special cue markers. Each segment is represented by a set of predefined features (e.g. location of the segment, average term frequencies of the words occurring in the segment, number of title words in the segment, and the like). Then a supervised learning algorithm is used to train the summarizer to extract important sentence segments, based on the feature vector. Results of experiments on U.S. patents indicate that the performance of the proposed approach compares very favorably with other approaches (including Microsoft Word summarizer) in terms of precision, recall, and classification accuracy. Seonggi Ryang et al. [100] presented a new approach to the problem of automatic 
text summarization called ASRL (Automatic Summarization using Reinforcement Learning ). In this paper, which models the process of constructing a summary with the framework of reinforcement learning and attempts to optimize the given score function with the given feature representation. The experimental results demonstrated ASRL tends to converge sub-optimally, and excessively depends on the formulation of features and the score function.

\subsection{Query based summarization}

Query based text summarization gives the right volume of the required information according to search query given by the person. Hence, the user does not need to invest extensive time for searching required information. In this summarization method, the sentences in a known document are scored based on a query using criteria such as frequency counts of terms. Those sentences comprising the query expressions are given higher scores than the ones containing fewer query words. Then, the sentences having maximum scores are merged into the output summary. Query based text summarization gives accurate results. If a query contains only little terms, this may cause important information loss in summary. Tingting He et al. [38 proposed a feature fusion based sentence selecting strategy, to identify the sentences with high query-relevance and high information density. The model scores each sentence by computing its similarity and Skip-Bigram co-occurrence with the query. These two features can measure the query-relevance from content and structure respectively. Then, they re-score the sentences using the information density feature gained from a text graph which can provide position information. Moreover, finally, they adopt MMR 2 for sentence extracting.Lu Wang et al.[114] proposed a submodular function-based opinion summarization framework. Tested on community QA and blog summarization, this approach outperforms state-of-the-art methods that are also based on submodularity in both automatic evaluation and human evaluation. The framework is capable of including statistically learned sentence relevance and encouraging the summary to cover diverse topics. A study on different metrics on text similarity estimation and their effect on summarization have been performed. Query-expansion is an effective Relevance Feedback technique for improving performance in Information Retrieval. Motivated by the hypothesis that query expansion terms should only be sought from the most relevant areas of a document, this investigation explores the use of document summaries in query-expansion. The investigation explores the use of both context-independent standard summaries and query-biased summaries. The paper also presents a novel approach to term-selection that separates the choice of relevant documents from the selection of a pool of potential expansion terms. Adenike M. Lam-Adesina et al.[56] have reported an investigation into the use of document summarization for term-selection in pseudo- relevance feedback. Summarization has been shown to be effective in this application with query-biased summaries potentially slightly better than context-independent summaries. Gabriel Murray et al. [79] Though the LSA method consistently performed the best, it was not a significant improvement over MMR and does not share some of the advantages of MMR. For example, MMR is ideal for query-based and multi-document summarization, and this work wants users to be able to create query-based summaries of meetings they were unable to attend. This work is preliminary in that it relies on a very small prosodic database. Dragomir Radev et al.

\footnotetext{
${ }^{2}$ Maximal marginal relevance is an important "diversity based ranking technique", used to maximizes the relevance and novelty in finally retrieved top-ranked items
}

[95] describe the functionality of MEAD, a comprehensive, public domain, open source, multi document multilingual summarization environment that has been thus far downloaded by more than 500 organizations. MEAD has been used in a variety of summarization applications ranging from summarization for mobile devices to Web page summarization within a search engine and to novelty detection. MEAD has been successfully used to evaluate an existing summarizer, to test a summarization feature, to test a new evaluation metric, test a short-query machine translation system. 1lias Chali et al. [37| applied sentence compression models for the task of query-focused multi-document summarization to investigate if sentence compression improves the overall summarization performance. Both compression and summarization are considered as global optimization problems and solved using integer linear programming (ILP). Three different models are built depending on the order in which compression and summarization are performed: 1) ComFirst (where compression is performed first), 2) SumFirst (where important sentence extraction is performed first), and 3) Combined (where compression and extraction are performed jointly via optimizing a combined objective function). Sentence compression models include lexical, syntactic and semantic constraints while summarization models include relevance, redundancy and length constraints. A comprehensive set of query-related and importance-oriented measures are used to define the relevance constraint whereas four alternative redundancy constraints are employed based on different sentence similarity measures using a) cosine similarity, b) syntactic similarity, c) semantic similarity, and d) extended string subsequence kernel (ESSK). Empirical evaluation on the DUC benchmark datasets demonstrates that the overall summary quality can be improved significantly using global optimization with semantically motivated models. Vivi Nastase [82] demonstrated that producing a good summary of the relevant information relies on understanding the query and linking it with the associated set of documents The experiments conducted within the summarization framework of the Document Understanding Conference have confirmed that encyclopedic knowledge extracted from Wikipedia can benefit the summarization task. Wikipedia articles are a source of relevant related concepts that are useful for expanding a summarization query. Tsutomu HIRAO et al. [42] presented a Question-Biased Text Summarization approach in which the Hanning window is applied to a Question Answering task. Summarization experiments were conducted on the lead based method, a tf * idf based method, and a Hanning window-based method.

\section{ABSTRACTIVE TEXT SUMMARIZATION METHOD}

Abstractive summarization creates a generalized summary by constructing new sentences alike a human being which is short and concise. Summary may contain new sentences that are not available in the source text. For generating abstractive summary language generation(NLG i. e Natural Language Generation) and compression techniques are necessary. Abstractive text summarization broadly catagorised into two types: Structure based and Semantic based approach.

\subsection{Structure Based Approach}

In Structure based approach the most important information from the document is analyssed through cognitive schemas viz tree, ontology, lead and body phrase structure.

3.1.1 Tree Based Method. Tree based method represents the text sentences in a dependency tree. Source text is first represented in 
the form of dependency trees then these tree are consolidating in a single tree and finally the merged dependency tree is converted to a sentence which is known as the fused sentence. The process of converting a dependency tree into a string of words is called as tree linearization. The performance of tree based summarization mainly depend upon on the parser chosen and dependency preserved between words. Yimai Fang et al. [21] proposed a first prototype of the feasibility of basing a summarization algorithm on Kintsch et al. (1978) model. It creates flexible-length summaries. It discriminates between $\mathrm{n}$ long-term memory (LTM) and short-term memory (STM) to two parallel salience levels from KvDs (Kintsch and van Dijk ) two disjoint stages, formalized the tree building process and improved KvDs root choice strategy. Anything that improves the proposition builder should bear direct fruit in the quality of the summaries. The limitations of this technology is semantics is not fully determined by syntax. Dan Gillick et al. [28] synthesized a number of ideas from the field of automatic summarization, including concept-level weighting, a maximum coverage model to minimize redundancy globally, and sentence compression derived from parse trees. While an ILP formulation for summarization is not novel, this method provides reasonably scalable, efficient solutions for practical problems, including those in recent TAC and DUC evaluations. Mehdi Yousfi-Monod et al. [117] addressed the task of sentence compression based on a deep linguistic analysis. The system is called COLIN. It relies on a parts of the sentence and dependencies of sentence tree pruning which removes those branches which could be cut without jeopardizing the sentence construction, or tempering too strongly with the sentence meaning. A careful study of syntactic properties, lexical functions, verbs arguments has led to design several different configurations in which the sentence compression quality could degrade if compression goes too far. The appreciation of a compression quality has been here demonstrated as a user satisfaction protocol. Fei Liu et al. [63] presented a novel abstractive summarization framework that draws on the recent development of a treebank for the Abstract Meaning Representation (AMR). In this the source text is parsed to a set of AMR graphs, the graphs are converted into a summary graph, and then text is generated from the summary graph. The paper focuses on the graph-to-graph transformation that reduces the source semantic graph into a summary graph, making use of an existing AMR parser and assuming the eventual availability of an AMRto-text generator. The framework is data-driven, trainable, and not specifically designed for a particular domain. Experiments on gold standard AMR annotations and system parses show good results.

3.1.2 Template Based Method. Here Template is used represent the document. Text is matched to patterns and rules to distinguish text content that mapped into template space. Template based systems differ in linguistic coverage, syntactic acquaintance, and steps involved in filling the templates. Text which fits into template shows the content of summary. This type summary generated is highly coherent. Templates has relevant content and it requires detailed semantic analysis hence it is the main problem faced by template based method. Harabagiu et al. [36] uses a template to extract information from multiple text documents. Ad hoc template are filled with snippets from multiple documents that follow pattern and rules defined to generate summary. Embar et al. [19] proposed a system which uses abstraction scheme with domain knowledge template containing IR rules. Number of a templates are created with variety of forms to create summary. Oya et al. [90] use important phrases from meeting transcript. Abstractive summary is generated by filling topic portion into appropriate template. Zhang et al. [121] recognize speech act to fill the template with keywords to generate template based abstractive summary. Speech act recognized with word feature, symbol based feature. Tweets are ranked based on n-gram occurrence of topic words and salient words to generate an abstractive summary.

3.1.3 Ontology Based Method. Ontology is a formal naming and definition of the entity types that are related to particular domain act as a knowledge base. In this method, a knowledge base is used to improve summarization result. Most documents on the internet are related to a particular domain having a limited vocabulary that can be better represented by the ontology. With the help of ontology attributes we can improve the semantic representation of information content and query expansion. Leonhard Hennig et al. [40] describe how sentences can be mapped to nodes of a flexible, widecoverage ontology. It shows that the mapping provides a semantic representation of the information content of sentences that improves summarization quality. From the category labels themselves as well as from the structural properties of the taxonomy it computes various sentence features which improve the accuracy of an SVM classifier trained on the task of sentence classification. Furthermore, this work provides experimental results which shows that Rouge scores of summaries generated from the classification output of an SVM trained with ontology-based sentence features outperform summaries generated from an SVM trained only on standard features from summarization research. Lee et al.[58] presented a fuzzy system that uses ontology designed by News domain expert. Sentences are classified according to term classifier which uses ontology. The fuzzy inference mechanism calculates membership degree for each sentence according to term classifier based on domain ontology. Raghunath et al. [97 proposed ontology based summarization that uses concept term, feature vector. They encoded ontology with tree structure node representing concept. The hierarchical classifier will select sentence according to tree structured ontology to generate summary.Hennig et al. [40] map sentences to nodes of ontology attributes. SVM classifier is trained on the binary feature. A bag of tag label is associated with each sentence represent dot product of two feature vector represents information in ontology space. Sentence of Leaf node of subtree contains specific information and classified as summary sentence. Baralis et al. [4] analyze the document to map words on yago ontology entities and calculate entity relevance score to rank sentences. Iteratively re-ranking is done to select top sentences to generate summary.

3.1.4 Lead and Body Phrase Method. This method is based on phrases. In lead and body phrase method main sentences, i.e. sentences which are informative in context and have good length are rephrase by inserting and substituting phrase. This method is good for semantically appropriate revisions for revising a lead sentence. One of the major drawbacks of Lead and body phrase is parsing degrade the performance and no generalized model for summarization. Mark Wasson [115] Leading text extracts created to support some online Boolean retrieval goals are evaluated for their acceptability as news document summaries. The results of this investigation show that leading text can provide acceptable summaries for most general news documents. These results are consistent with Brandow et al. (1995). Leading text extracts also have two less obvious advantages over other approaches. First, legal restrictions often prevent us from manipulating copyrighted material. Leading text extracts often preserve the existing copyright. Second, when leading text fails as a summary, customers can see why. Customer understanding of how a data feature is created is often key to customer acceptance of that feature. Lin et al. (1997) describe methods for identifying the likely locations of topic-bearing sentences. 
Comparing the content of leading text extracts to predictions of topic-bearing sentences may help to predict where leading text fails as a summary so that one can direct more sophisticated approaches to those documents. Jahna Otterbacher et al. [89] present an evaluation of a novel hierarchical extractive text summarization method that allows users to view summaries of Web documents from small, mobile devices. The method was tested in the context of a Web mail system, which allows a user to access his or her inbox, to which a set of news articles has been sent. The method also tested the use of hierarchical summaries against the use of full text documents as well as three baseline summarization methods (top 20\%, lead-based and random) on the task of finding answers to questions about the given news stories. It was found that there was no significant difference in terms of task accuracy and completion times between the full text document and hierarchical summarization settings. In addition, the use of the hierarchical summaries reduces the number of bytes per user request by more than half. Tanaka et al.[108] search for the same chunk in lead body sentence called triggers. Phrases are identified according to similarity for substitution of body phrase into the lead phrase. This process is done iteratively to generate new summary sentences. Ishikawa et al.[45] proposed hybrid summarization method based TF method and LEAD method. Rectangular distribution function multiplied with term frequency which assigns weight to every sentence to identify importance. Sentences are ranked according to importance to compose summary.

3.1.5 Rule Based Method. In rule based method content selection is done with the help of information extraction rules explicitly specified by the user. Finally, language patterns are used for generating summary sentences. The strong point of this method is it creates summaries with greater information density. The main drawback is that all the rules and patterns are manually written, which is a tedious and time consuming task. Pierre-Etienne Genest et al. [27] showed that full abstraction can be accomplished in the context of guided summarization and describes a work in progress that relies on Information Extraction, statistical content selection and Natural Language Generation. Early results already demonstrate the effectiveness of the approach. The higher density of information in our short summaries is one key to address the performance ceiling of extractive summarization methods. They proposed abstraction scheme consist of information extraction rules by identifying noun and verb based on scheme event. The Heuristic is used to select an appropriate sentence. Patterns are designed with Simple Natural Language Generation rules to generate summary with less redundancy. Sankar K et al. [102] propose a coherence chunker module and a hash based approach to graph based ranking algorithm for text ranking. In specific, they propose a novel approach for graph based text ranking, with improved results comparative to existing ranking algorithms. The architecture of the algorithm helps the ranking process to be done in a time efficient way. This approach succeeds in grabbing the coherent sentences based on the linguistic and heuristic rules; whereas other supervised ranking systems do this process by training the summary collection. This makes the proposed algorithm highly portable to other domains and languages. Filippo Galgani et al. [24] this paper describes a hybrid approach in which a number of different summarization techniques are combined in a rule-based system using manual knowledge acquisition, where human intuition, supported by data, specifies not only attributes and algorithms, but the contexts where these are best used. We apply this approach to automatic summarization of legal case reports.

\subsection{SEMANTIC BASED APPROACH}

In Semantic based method, semantic representation of the document is given to natural language generation (NLG) system. This method focuses on identifying noun and verb phrases by processing linguistic data. Following are the methods of Semantic Based Approach.

3.2.1 Multimodal semantic model. In Multimodal method semantic model is constructed to capture link among concepts. The important concepts are scored based on measure and selected concept is represented as summary. The Key point of this method is coverage of information content. Greenbacker [31] proposed approach which works in three stages first it uses an ontology to build a semantic model which represents the multimodal document. Second with information density matrix which rates a concept based on a factor such as completeness of attribute, the number of connections. Information density matrix is use to score concept and finally, summary is generated with high score concept.

3.2.2 Information item based method. In this method, a summary is generated from the abstract representation of source document. The information item is the smallest element of coherent information in a text. Information item based method provides less redundant and concise summaries. Pierre-Etienne Genest et al.[26] propose a new, ambitious framework for abstractive summarization, which aims at selecting the content of a summary not from sentences, but from an abstract representation of the source documents. This abstract representation relies on the concept of Information Items which is defined as the smallest element of coherent information in a text or a sentence. They proposed framework having information item retrieval, sentence generation, sentence selection and summary generation. In analysis part subjectverbobject (SVO) triplet extracted. Sentence are generated using Simple NLG realize. The Sentence is ranked based on document frequency and summary is generated.

3.2.3 Semantic Graph Based Method. In semantic graph method, the input document is semantically represented using semantic graph. Noun and verb from the sentences are represented as graph nodes and relation between them is given by edge. It produces concise, coherent and less redundant and grammatically correct sentences. Moawad et al. [78]constructed a semantic graph called rich semantic graph to represent the semantic of a source document. Sentence ranking is done based on deriving the average weight of word and sentence. With highest rank sentence Rich Semantic Graph is generated and graph reduction is performed with heuristic rules to generate an abstractive summary. Meru Brunn et al. [8] presented an efficient implementation of the lexical cohesion approach as the driving engine of the summarization system. The ranking procedure, which handles the text aboutness measure, is used to select the most salient and best connected sentences in a text corresponding to the summary ratio requested by the user. In the future, we plan to investigate the following problems: Our methods extract whole sentences as single units. The use of compression techniques will increase the condensation of the summary and improve its quality (Barzilay, McKweon, and Elhadad, 1999; Mani, Gates, and Bloedorn, 1999; Jing and McKeown, 2000; Knight and Marcu, 2000). The summarization method uses only lexical chains as representations of the source text. Other clues could be gathered from the text and considered when generating the summary. In the noun filtering process, our hypothesis eliminates the terms in subordinate clauses. Rather than eliminating them, it may also prove fruitful to investigate weighting terms according to the kind of clause 
in which they occur. H. Gregory Silber et al. [104] present a lineartime algorithm for lexical chain computation. The algorithm is used for computing lexical chains as an intermediate representation for automatic machine text summarization that it uses the method proposed by Barzilay and Elhadad, but it is clearly $\mathrm{O}(\mathrm{n})$ in the number of nouns in the source document. The benefit of this linear-time algorithm is its ability to compute lexical chains in documents significantly larger than could be handled by Barzilay and Elhadads implementation. Doina Tatar et al. [109] showed that the text segmentation by lexical chains and by text entailment relation between sentences are good bases for obtaining highly accurate summaries. Moreover, the method replaces the usually bottom-up lexical chain construction with a top-down one, where first a single chain of disambiguated words is established and then it is divided in a sequence of many shorter lexical chains. The segmentation of text follows the sequence of lexical chains. The methods of summarization control the length of the summaries by a process of scoring the segments. Thus, more material is extracted from the strongest segments. Regina Barzilay et al. [5] introduced method called Lexical chains. It uses the WordNet database for determining cohesive relations (i.e., repetition, synonymy, antonymy, hypernymy, and holonymy) between terms. The chains are then composed by related terms. Their scores are determined on the basis of the number and type of relations in the chain. Sentences where the strongest chains are highly concentrated are selected for the summary.

\subsection{Abstractive Text Summarization using Neural Network}

Abstractive text summarization is a complex task whose goal is to generate a concise version of a text without necessarily reusing the sentences from the original source, but still preserving the meaning and the key contents. Research of Ramesh Nallapati et al.[81] models an abstractive text summarization using Attentional EncoderDecoder RNN, and shows that they achieve state-of-the-art performance on two different corpora. Each of the proposed novel models addresses a specific problem in abstractive summarization, yielding further improvement in performance. The paper proposes a new dataset for multi-sentence summarization and establishes benchmark numbers on it. Alexander M. Rush et. al.[99] utilizes a local attention-based model that generates each word of the summary conditioned on the input sentence. This work is based on recent developments in neural machine translation. They combine probabilistic model with a generation algorithm which produces accurate abstractive summaries. While the model is structurally simple, it can easily be trained end-to-end and scales to a large amount of training data. Sumit Chopra et al.[14] introduced a conditional recurrent neural network (RNN) which generates a summary of an input sentence. The conditioning is provided by a novel convolutional attention-based encoder which ensures that the decoder focuses on the appropriate input words at each step of generation. The model relies only on learned features and is easy to train in an end-to-end fashion on large data sets. Gaetano Rossiello et. al.[98] outlined our ongoing research on abstractive text summarization using deep learning models. The abstractive summarization is a harder task than extractive summarization, where the techniques produce a summary by selecting the most relevant sentences from an input source text. They propose a novel approach to combine probabilistic models with neural networks in a unified way in order to incorporate prior knowledge such as linguistic features. Shujian Liu[66]focus on using neural networks techniques and statistical language models for abstractive summarization on long texts. Results have shown that picking shortest-clause from most impor- tant sentence choosen by LexRank Algorhtm appears to have the best performance. Lu Wang et al.[113] propose an attention-based neural network model that is able to absorb information from multiple text units to construct informative, concise, and fluent summaries. An importance-based sampling method is designed to allow the encoder to integrate information from an important subset of input. They presented a neural approach to generate abstractive summaries for opinionated text. They employed an attentionbased method that finds salient information from different input text units to generate an informative and concise summary. To cope with the large number of input text, we deploy an importancebased sampling mechanism for model training. Karl Moritz Hermann et. al.[41] demonstrated a methodology for obtaining a large number of document-query answer triples and shown that recurrent and attention based neural networks provide an effective modelling framework for this task. The supervised paradigm for training machine reading and comprehension models provides a promising avenue for making progress on the path to building full natural language understanding systems. The analysis indicates that the Attentive and Impatient Readers are able to propagate and integrate semantic information over long distances. In particular we believe that the incorporation of an attention mechanism is the key contributor to these results. Urvashi Khandelwal[52] attempted to build a neural network based text summarization model that could operate on a single domain scientific publication corpus. They modified training to use imitation learning where the model has to make a decision between using the model generated output vs. the gold token at each time step. The expectation was that this would make minimizing the loss very hard and would push the model to use its source representation. Learning a neural summarization model on a dataset of limited size is a hard task.

\section{TEXT SUMMARIZATION EVALUATION METHODS}

Evaluating a summary is a difficult and essential task because there does not exist an ideal summary for a given document or set of documents. It is not easy for humans to know what kind of information should be present in the summary. Information changes according to the purpose of the summary and to capture this information automatically, is a difficult task. Therefore, reliable automatic evaluation metrics are required for fast and consistent evaluation. Methods for evaluating systems can be broadly classified into two broad categories extrinsic and intrinsic.

\subsection{Extrinsic evaluation}

It determines summarys quality based on how it affects other tasks (Text classification, Information retrieval, Question answering), i.e., a summary is termed as a good summary if it provides help to other tasks. Various methods for extrinsic evaluation are

-Relevance assessment: Here various methods are used for evaluating a topics relevance present in the summary or the original document.

- Reading comprehension: It determines whether it is able to answer multiple choice tests after reading the summary.

\subsection{Intrinsic evaluation}

-Quality: It determines the summary quality on the basis of coverage between the machine-made summary and the human-made summary. 
- The informativeness of a summary is evaluated by comparing it with a human-made summary, i.e., reference summary. Quality or informativeness are the two important aspects on the basis of which a summary is evaluated.

-Fidelity to the source which checks whether the summary consists of the same or similar content as present in the original document.

The main evaluation metrics of co-selection are precision, recall and F-score. Precision (P) is the number of sentences occurring in both system and ideal summaries divided by the number of sentences in the system summary. Recall (R) is the number of sentences occurring in both system and ideal summaries divided by the number of sentences in the ideal summary. F-score is a composite measure that combines precision and recall. The basic way how to compute the F-score is to count a harmonic average of precision and recall

$$
F=\frac{2 P R}{P+R}
$$

An assessment of a summary can be done in different ways. Several examples, like Shannon Game or Question Game can be found in [62]. In summary evaluation programs such as SUMMAC or DUC automatic generated summaries (extracts or abstract) are evaluated mostly intrinsically against human reference or gold-standard summaries (ideal summaries). The problem is to establish what an ideal summary is. Humans know how to sum up the most important information of a text. However, different experts may disagree in considering which information is the best to be extracted. Automatic evaluation programs have therefore been developed to try to give an objective point of view of evaluation. Systems like SEE(Summarization Evaluation Environment), ROUGE( RecallOriented Understudy for Gisting Evaluation ) or BE(Basic Elements)or BLEU ${ }^{3}$ have been created to help to this task. The Pyramid method is a novel semi-automatic evaluation method [108]. Its basic idea is to identify summarization content units (SCUs) that are used for comparison of information in summaries. Lin [59] introduced a set of metrics called Recall-Oriented Understudy for Gisting Evaluation (ROUGE) that have become standards of automatic evaluation of summaries. Jun-Ping $\mathrm{Ng}$ et al. [85] adopted ROUGE automatic evaluation measure for text summarization. While it has been shown to correlate well with human judgements, it is biased towards surface lexical similarities. This makes it unsuitable for the evaluation of abstractive summarization, or summaries with substantial paraphrasing. Feifan Liu et al. [64] made a first attempt to systematically investigate the correlation of automatic ROUGE scores with human evaluation for meeting summarization. Adaptations on ROUGE setting based on meeting characteristics are proposed and evaluated using Spearmans rank coefficient. The experimental results show that in general the correlation between ROUGE scores and human evaluation is low, with ROUGE SU4 score showing better correlation than ROUGE1 score. There is significant improvement in correlation when disfluencies are removed and speaker information is leveraged, especially for evaluating system-generated summaries. It is observed that the correlation is affected differently by those factors for human summaries and system generated summaries. Horacio Saggion et al. [101] explain correlation of rankings of text summarization systems using evaluation methods with and without human models.

\footnotetext{
${ }^{3} \mathrm{BLEU}$ (bilingual evaluation understudy) is an algorithm for evaluating the quality of text which has been machine-translated from one natural language to another.
}

It applies comparison framework to various well-established content based evaluation measures in text summarization such as coverage, Responsiveness, Pyramids and ROUGE studying their associations in various text summarization tasks including generic and focus-based multi-document summarization in English and generic single-document summarization in French and Spanish. Chin-Yew Lin et al. [62] gave a brief introduction of the manual summary evaluation protocol used in the Document Understanding Conference. The paper discusses the IBM BLEU MT evaluation metric, its application to summary evaluation, and the difference between precision based BLEU translation evaluation and recall-based summary evaluation. The paper proposes two test criteria for evaluation. One was to make sure system rankings produced by automatic scoring metrics were similar to human rankings. This was quantified by Spearmans rank order correlation coefficient and three other parametric correlation coefficients. Another was to compare the statistical significance test results between automatic scoring metrics and human assessments. The recall and precision of the agreement between the test statistics results to identify good automatic scoring metrics is used. According to the experiments, it is found that unigram co-occurrence statistics is a good automatic scoring metric. It consistently correlated highly with human assessments and had high recall and precision in significance test with manual evaluation results. Josef Steinberger et al. [105] designed a measure is covered by Latent Semantic Analysis (LSA) which can capture the main topics of a document. The summarization systems are ranked according to the similarity of the main topics of their summaries and their reference documents. Results show a high correlation between human rankings and the LSA-based evaluation measure. The measure is designed to compare a summary with its full text. It can compare a summary with a human written abstract as well; however, in this case using a standard ROUGE measure gives more precise results. Nevertheless, if abstracts are not available for a given corpus, using the LSA-based measure is an appropriate choice. Inderjeet Mani et al. [70,74, 73] developed TIPSTER Text Summarization Evaluation (SUMMAC) that has established several new extrinsic and intrinsic methods for evaluating summaries. SUMMAC has recognized definitively in a large-scale evaluation that automatic text summarization is very effective in relevance assessment tasks on newspaper articles. . In recent years, new areas such as multi-document summarization and multi-lingual summarization have assumed increasing importance, posing new requirements for evaluations. New applications for summarization, such as question-answering, condensation and navigation of book-length materials, summaries for hand-held devices, etc., will create new opportunities as well as challenges for summarization evaluation. Lin et al. [61] describe and compare various human and automatic metrics to evaluate summaries. They focus on the evaluation procedure used in the Document Understanding Conference 2001 (DUC2001), where the Summary Evaluation Environment (SEE) interface was used to support the human evaluation part.

\section{CONCLUSION}

This survey paper covers extractive, abstractive summarization and evaluation techniques. Summarization system should produce an effective summary in a short time with less redundancy having grammatically correct sentences. Both extractive and abstractive method yields good result according to the context in which they used. The reviewed literature opens up the challenging area for hybridization of these methods to produce informative, well compressed and readable summaries. Various methods for evaluating summarization systems are discussed. 


\section{REFERENCES}

[1] Rafeeq Al-Hashemi. Text summarization extraction system (tses) using extracted keywords. Int. Arab J. e-Technol., 1(4):164-168, 2010.

[2] Ramiz M Aliguliyev. A new sentence similarity measure and sentence based extractive technique for automatic text summarization. Expert Systems with Applications, 36(4):77647772, 2009.

[3] Massih-Reza Amini and Patrick Gallinari. The use of unlabeled data to improve supervised learning for text summarization. In Proceedings of the 25th annual international ACM SIGIR conference on Research and development in information retrieval, pages 105-112. ACM, 2002.

[4] Elena Baralis, Luca Cagliero, Saima Jabeen, Alessandro Fiori, and Sajid Shah. Multi-document summarization based on the yago ontology. Expert Systems with Applications, 40(17):6976-6984, 2013.

[5] Regina Barzilay and Michael Elhadad. Using lexical chains for text summarization. Advances in automatic text summarization, pages 111-121, 1999.

[6] Phyllis B Baxendale. Machine-made index for technical literaturean experiment. IBM Journal of Research and Development, 2(4):354-361, 1958.

[7] Wauter Bosma and VU CLTL. Contextual salience in querybased summarization. In RANLP, pages 39-44, 2009.

[8] Meru Brunn, Yllias Chali, and Christopher J Pinchak. Text summarization using lexical chains. In Proc. of Document Understanding Conference, 2001.

[9] Orkut Buyukkokten, Hector Garcia-Molina, and Andreas Paepcke. Seeing the whole in parts: text summarization for web browsing on handheld devices. In Proceedings of the 10th international conference on World Wide Web, pages 652-662. ACM, 2001.

[10] Praveen Bysani. Detecting novelty in the context of progressive summarization. In Proceedings of the NAACL HLT 2010 Student Research Workshop, pages 13-18. Association for Computational Linguistics, 2010.

[11] Asli Celikyilmaz and Dilek Hakkani-Tur. A hybrid hierarchical model for multi-document summarization. In Proceedings of the 48th Annual Meeting of the Association for Computational Linguistics, pages 815-824. Association for Computational Linguistics, 2010.

[12] Hakan Ceylan and Rada Mihalcea. Explorations in automatic book summarization. Association for Computational Linguistics, 2007.

[13] Kuo-En Chang, Yao-Ting Sung, and Ine-Dai Chen. The effect of concept mapping to enhance text comprehension and summarization. The Journal of Experimental Education, 71(1):5-23, 2002.

[14] Sumit Chopra, Michael Auli, Alexander M Rush, and SEAS Harvard. Abstractive sentence summarization with attentive recurrent neural networks. In HLT-NAACL, pages 93-98, 2016.

[15] Wesley T Chuang and Jihoon Yang. Extracting sentence segments for text summarization: a machine learning approach. In Proceedings of the $23 \mathrm{rd}$ annual international ACM SIGIR conference on Research and development in information retrieval, pages 152-159. ACM, 2000.
[16] Dipanjan Das and André FT Martins. A survey on automatic text summarization. Literature Survey for the Language and Statistics II course at CMU, 4:192-195, 2007.

[17] Gerald DeJong. Prediction and substantiation: A new approach to natural language processing. Cognitive Science, 3(3):251-273, 1979 .

[18] Harold P Edmundson. New methods in automatic extracting Journal of the ACM (JACM), 16(2):264-285, 1969.

[19] Varsha R Embar, Surabhi R Deshpande, AK Vaishnavi, Vishakha Jain, and Jagadish S Kallimani. saramsha-a kannada abstractive summarizer. In Advances in Computing, Communications and Informatics (ICACCI), 2013 International Conference on, pages 540-544. IEEE, 2013.

[20] Günes Erkan and Dragomir R Radev. Lexrank: Graph-based lexical centrality as salience in text summarization. Journal of Artificial Intelligence Research, 22:457-479, 2004.

[21] Yimai Fang and Simone Teufel. A summariser based on human memory limitations and lexical competition. In EACL, pages 732-741, 2014.

[22] Atefeh Farzindar and Guy Lapalme. Legal text summarization by exploration of the thematic structures and argumentative roles. In Text Summarization Branches Out Workshop held in conjunction with ACL, pages 27-34, 2004.

[23] Rafael Ferreira, Luciano de Souza Cabral, Rafael Dueire Lins, Gabriel Pereira e Silva, Fred Freitas, George DC Cavalcanti, Rinaldo Lima, Steven J Simske, and Luciano Favaro. Assessing sentence scoring techniques for extractive text summarization. Expert systems with applications, 40(14):5755-5764, 2013.

[24] Filippo Galgani, Paul Compton, and Achim Hoffmann. Combining different summarization techniques for legal text. In Proceedings of the Workshop on Innovative Hybrid Approaches to the Processing of Textual Data, pages 115123. Association for Computational Linguistics, 2012.

[25] Kavita Ganesan, ChengXiang Zhai, and Jiawei Han. Opinosis: a graph-based approach to abstractive summarization of highly redundant opinions. In Proceedings of the 23 rd international conference on computational linguistics, pages 340-348. Association for Computational Linguistics, 2010.

[26] Pierre-Etienne Genest and Guy Lapalme. Framework for abstractive summarization using text-to-text generation. In Proceedings of the Workshop on Monolingual Text-To-Text Generation, pages 64-73. Association for Computational Linguistics, 2011.

[27] Pierre-Etienne Genest and Guy Lapalme. Fully abstractive approach to guided summarization. In Proceedings of the 50th Annual Meeting of the Association for Computational Linguistics: Short Papers-Volume 2, pages 354-358. Association for Computational Linguistics, 2012.

[28] Dan Gillick and Benoit Favre. A scalable global model for summarization. In Proceedings of the Workshop on Integer Linear Programming for Natural Langauge Processing, pages 10-18. Association for Computational Linguistics, 2009.

[29] Jade Goldstein, Mark Kantrowitz, Vibhu Mittal, and Jaime Carbonell. Summarizing text documents: sentence selection and evaluation metrics. In Proceedings of the 22nd annual international ACM SIGIR conference on Research and development in information retrieval, pages 121-128. ACM, 1999. 
[30] Yihong Gong and Xin Liu. Generic text summarization using relevance measure and latent semantic analysis. In Proceedings of the 24th annual international ACM SIGIR conference on Research and development in information retrieval, pages 19-25. ACM, 2001.

[31] Charles F Greenbacker. Towards a framework for abstractive summarization of multimodal documents. In Proceedings of the ACL 2011 Student Session, pages 75-80. Association for Computational Linguistics, 2011.

[32] Anand Gupta, Manpreet Kaur, Adarsh Singh, Aseem Goel, and Shachar Mirkin. Text summarization through entailment-based minimum vertex cover. Lexical and Computational Semantics (* SEM 2014), page 75, 2014.

[33] Vishal Gupta and Gurpreet Singh Lehal. Named entity recognition for punjabi language text summarization. International journal of computer applications, 33(3):28-32, 2011.

[34] Aria Haghighi and Lucy Vanderwende. Exploring content models for multi-document summarization. In Proceedings of Human Language Technologies: The 2009 Annual Conference of the North American Chapter of the Association for Computational Linguistics, pages 362-370. Association for Computational Linguistics, 2009.

[35] Udo Hahn and Inderjeet Mani. The challenges of automatic summarization. Computer, 33(11):29-36, 2000.

[36] Sanda M Harabagiu and Finley Lacatusu. Generating single and multi-document summaries with gistexter. In Document Understanding Conferences, pages 11-12, 2002.

[37] Yllias Chali Sadid A Hasan. On the effectiveness of using sentence compression models for query-focused multidocument summarization. 2012.

[38] Tingting He, Fang Li, Wei Shao, Jinguang Chen, and Liang Ma. A new feature-fusion sentence selecting strategy for query-focused multi-document summarization. In Advanced Language Processing and Web Information Technology, 2008. ALPIT'08. International Conference on, pages 81-86. IEEE, 2008.

[39] Leonhard Hennig and DAI Labor. Topic-based multidocument summarization with probabilistic latent semantic analysis. In $R A N L P$, pages 144-149, 2009.

[40] Leonhard Hennig, Winfried Umbrath, and Robert Wetzker. An ontology-based approach to text summarization. In Proceedings of the 2008 IEEE/WIC/ACM International Conference on Web Intelligence and Intelligent Agent TechnologyVolume 03, pages 291-294. IEEE Computer Society, 2008.

[41] Karl Moritz Hermann, Tomas Kocisky, Edward Grefenstette, Lasse Espeholt, Will Kay, Mustafa Suleyman, and Phil Blunsom. Teaching machines to read and comprehend. In $A d$ vances in Neural Information Processing Systems, pages 1693-1701, 2015.

[42] Tsutomu Hirao, Yutaka Sasaki, and Hideki Isozaki. An extrinsic evaluation for question-biased text summarization on qa tasks. In Proc. of the NAACL 2001 Workshop on Automatic Summarization, pages 61-68, 2001.

[43] Baotian Hu, Qingcai Chen, and Fangze Zhu. Lcsts: A large scale chinese short text summarization dataset. arXiv preprint arXiv:1506.05865, 2015.

[44] Po Hu, Dong-Hong Ji, Chong Teng, and Yujing Guo. Context-enhanced personalized social summarization. In COLING, pages 1223-1238, 2012.
[45] Kai Ishikawa, Shinichi Ando, and Akitoshi Okumura. Hybrid text summarization method based on the tf method and the lead method. In In Proceedings of the 2nd National Institute of Informatics Test Collection Information Retrieval (NTCIR) Workshop. Citeseer, 2001.

[46] R Jayashree, K Srikanta Murthy, and Basavaraj S Anami. An artificial neural network approach to text document summarization in the kannada language. In Hybrid Intelligent Systems (HIS), 2013 13th International Conference on, pages 45-48. IEEE, 2013.

[47] R Jayashree, Srikanta Murthy, and Basavaraj S Anami. Categorized text document summarization in the kannada language by sentence ranking. In Intelligent Systems Design and Applications (ISDA), 2012 12th International Conference on, pages 776-781. IEEE, 2012.

[48] Hongyan Jing. Sentence reduction for automatic text summarization. In Proceedings of the sixth conference on Applied natural language processing, pages 310-315. Association for Computational Linguistics, 2000.

[49] Mikael Kågebäck, Olof Mogren, Nina Tahmasebi, and Devdatt Dubhashi. Extractive summarization using continuous vector space models. In Proceedings of the 2nd Workshop on Continuous Vector Space Models and their Compositionality (CVSC)@EACL, pages 31-39, 2014.

[50] Khosrow Kaikhah. Automatic text summarization with neural networks. In Intelligent Systems, 2004. Proceedings. 2004 2nd International IEEE Conference, volume 1, pages 40-44. IEEE, 2004.

[51] Chris Kedzie, Kathleen McKeown, and Fernando Diaz. Predicting salient updates for disaster summarization. In $A C L$ (1), pages 1608-1617, 2015.

[52] Urvashi Khandelwal. Neural text summarization.

[53] F KIOUMARSI, HAMID KHOSRAVI, E ESLAMI, and MOHSEN DAVOUDI. Extraction-based text summarization using fuzzy. 2010.

[54] P Arun Kumar, K Praveen Kumar, T Someswara Rao, and P Krishna Reddy. An improved approach to extract document summaries based on popularity. In International Workshop on Databases in Networked Information Systems, pages 310-318. Springer, 2005.

[55] Julian Kupiec, Jan Pedersen, and Francine Chen. A trainable document summarizer. In Proceedings of the 18th annual international ACM SIGIR conference on Research and development in information retrieval, pages 68-73. ACM, 1995.

[56] Adenike M Lam-Adesina and Gareth JF Jones. Applying summarization techniques for term selection in relevance feedback. In Proceedings of the 24th annual international ACM SIGIR conference on Research and development in information retrieval, pages 1-9. ACM, 2001.

[57] Yulia Ledeneva, Alexander Gelbukh, and René Arnulfo García-Hernández. Terms derived from frequent sequences for extractive text summarization. In International Conference on Intelligent Text Processing and Computational Linguistics, pages 593-604. Springer, 2008.

[58] Chang-Shing Lee, Zhi-Wei Jian, and Lin-Kai Huang. A fuzzy ontology and its application to news summarization. IEEE Transactions on Systems, Man, and Cybernetics, Part B (Cybernetics), 35(5):859-880, 2005. 
[59] Chin-Yew Lin. Rouge: A package for automatic evaluation of summaries. In Text summarization branches out: Proceedings of the ACL-04 workshop, volume 8. Barcelona, Spain, 2004.

[60] Chin-Yew Lin and Eduard Hovy. The automated acquisition of topic signatures for text summarization. In Proceedings of the 18th conference on Computational linguistics-Volume 1, pages 495-501. Association for Computational Linguistics, 2000.

[61] Chin-Yew Lin and Eduard Hovy. Manual and automatic evaluation of summaries. In Proceedings of the ACL-O2 Workshop on Automatic Summarization-Volume 4, pages 45-51. Association for Computational Linguistics, 2002.

[62] Chin-Yew Lin and Eduard Hovy. Automatic evaluation of summaries using n-gram co-occurrence statistics. In Proceedings of the 2003 Conference of the North American Chapter of the Association for Computational Linguistics on Human Language Technology-Volume 1, pages 71-78. Association for Computational Linguistics, 2003.

[63] Fei Liu, Jeffrey Flanigan, Sam Thomson, Norman Sadeh, and Noah A Smith. Toward abstractive summarization using semantic representations. 2015.

[64] Feifan Liu and Yang Liu. Correlation between rouge and human evaluation of extractive meeting summaries. In Proceedings of the 46th Annual Meeting of the Association for Computational Linguistics on Human Language Technologies: Short Papers, pages 201-204. Association for Computational Linguistics, 2008.

[65] Shixia Liu, Michelle X Zhou, Shimei Pan, Yangqiu Song, Weihong Qian, Weijia Cai, and Xiaoxiao Lian. Tiara: Interactive, topic-based visual text summarization and analysis. ACM Transactions on Intelligent Systems and Technology (TIST), 3(2):25, 2012.

[66] Shujian Liu. Cs585 project report long text summarization using neural networks and rule-based approach. 2017.

[67] Xiaohua Liu, Yitong Li, Furu Wei, and Ming Zhou. Graphbased multi-tweet summarization using social signals. In COLING, volume 12, pages 1699-1714, 2012.

[68] Annie P Louis. A bayesian method to incorporate background knowledge during automatic text summarization. Association for Computational Linguistics, 2014.

[69] Hans Peter Luhn. The automatic creation of literature abstracts. IBM Journal of research and development, 2(2):159$165,1958$.

[70] Inderjeet Mani. Summarization evaluation: An overview. 2001.

[71] Inderjeet Mani and Eric Bloedorn. Multi-document summarization by graph search and matching. arXiv preprint cmplg/9712004, 1997.

[72] Inderjeet Mani, Eric Bloedorn, and Barbara Gates. Using cohesion and coherence models for text summarization. In Intelligent Text Summarization Symposium, pages 69-76, 1998.

[73] Inderjeet Mani, David House, Gary Klein, Lynette Hirschman, Therese Firmin, and Beth Sundheim. The tipster summac text summarization evaluation. In Proceedings of the ninth conference on European chapter of the Association for Computational Linguistics, pages 77-85. Association for Computational Linguistics, 1999.
[74] Inderjeet Mani, Gary Klein, David House, Lynette Hirschman, Therese Firmin, and Beth Sundheim. Summac: a text summarization evaluation. Natural Language Engineering, 8(01):43-68, 2002.

[75] Inderjeet Mani and Mark T Maybury. Advances in automatic text summarization, volume 293. MIT Press, 1999.

[76] Rada Mihalcea. Graph-based ranking algorithms for sentence extraction, applied to text summarization. In Proceedings of the ACL 2004 on Interactive poster and demonstration sessions, page 20. Association for Computational Linguistics, 2004.

[77] Rada Mihalcea and Paul Tarau. A language independent algorithm for single and multiple document summarization. In Proceedings of IJCNLP, volume 5, 2005.

[78] Ibrahim F Moawad and Mostafa Aref. Semantic graph reduction approach for abstractive text summarization. In Computer Engineering \& Systems (ICCES), 2012 Seventh International Conference on, pages 132-138. IEEE, 2012.

[79] Gabriel Murray, Steve Renals, and Jean Carletta. Extractive summarization of meeting recordings. 2005.

[80] NK Nagwani. Summarizing large text collection using topic modeling and clustering based on mapreduce framework. Journal of Big Data, 2(1):6, 2015.

[81] Ramesh Nallapati, Bowen Zhou, Caglar Gulcehre, Bing Xiang, et al. Abstractive text summarization using sequence-to-sequence rnns and beyond. arXiv preprint arXiv:1602.06023, 2016.

[82] Vivi Nastase. Topic-driven multi-document summarization with encyclopedic knowledge and spreading activation. In Proceedings of the Conference on Empirical Methods in Natural Language Processing, pages 763-772. Association for Computational Linguistics, 2008.

[83] Ani Nenkova and Annie Louis. Can you summarize this? identifying correlates of input difficulty for generic multidocument summarization. 2008.

[84] Ani Nenkova and Kathleen McKeown. A survey of text summarization techniques. In Mining text data, pages 43-76. Springer, 2012.

[85] Jun-Ping Ng and Viktoria Abrecht. Better summarization evaluation with word embeddings for rouge. arXiv preprint arXiv:1508.06034, 2015.

[86] Jun-Ping Ng, Yan Chen, Min-Yen Kan, and Zhoujun Li. Exploiting timelines to enhance multi-document summarization. In ACL (1), pages 923-933, 2014.

[87] Masaaki Nishino, Norihito Yasuda, Tsutomu Hirao, Shinichi Minato, and Masaaki Nagata. A dynamic programming algorithm for tree trimming-based text summarization. In HLT-NAACL, pages 462-471, 2015.

[88] Tadashi Nomoto and Yuji Matsumoto. Supervised ranking in open-domain text summarization. In Proceedings of the 40th Annual Meeting on Association for Computational Linguistics, pages 465-472. Association for Computational Linguistics, 2002.

[89] Jahna Otterbacher, Dragomir Radev, and Omer Kareem. News to go: hierarchical text summarization for mobile devices. In Proceedings of the 29th annual international ACM SIGIR conference on Research and development in information retrieval, pages 589-596. ACM, 2006. 
[90] Tatsuro Oya, Yashar Mehdad, and Raymond Ng. A templatebased abstractive meeting summarization: Leveraging summary and source text relationships. 2014.

[91] Chandra Prakash and Anupam Shukla. Human aided text summarizer" saar" using reinforcement learning. In Soft Computing and Machine Intelligence (ISCMI), 2014 International Conference on, pages 83-87. IEEE, 2014.

[92] Vahed Qazvinian and Dragomir R Radev. Scientific paper summarization using citation summary networks. In Proceedings of the 22nd International Conference on Computational Linguistics-Volume 1, pages 689-696. Association for Computational Linguistics, 2008.

[93] Vahed Qazvinian, Dragomir R Radev, and Arzucan Özgür. Citation summarization through keyphrase extraction. In Proceedings of the 23rd international conference on computational linguistics, pages 895-903. Association for Computational Linguistics, 2010.

[94] Dragomir Radev, Adam Winkel, and Michael Topper. Multi document centroid-based text summarization. In ACL 2002, 2002.

[95] Dragomir R Radev, Timothy Allison, Sasha BlairGoldensohn, John Blitzer, Arda Celebi, Stanko Dimitrov, Elliott Drabek, Ali Hakim, Wai Lam, Danyu Liu, et al. Mead-a platform for multidocument multilingual text summarization. In LREC, 2004.

[96] Dragomir R Radev, Hongyan Jing, and Malgorzata Budzikowska. Centroid-based summarization of multiple documents: sentence extraction, utility-based evaluation, and user studies. In Proceedings of the 2000 NAACL-ANLP Workshop on Automatic summarization, pages 21-30. Association for Computational Linguistics, 2000.

[97] R Ragunath and N Sivaranjani. Ontology based text document summarization system using concept terms. ARPN Journal of Engineering and Applied Sciences, 10(6):26382642, 2015.

[98] Gaetano Rossiello, Pierpaolo Basile, Giovanni Semeraro, Marco Di Ciano, and Gaetano Grasso. Improving neural abstractive text summarization with prior knowledge. 2016.

[99] Alexander M Rush, Sumit Chopra, and Jason Weston. A neural attention model for abstractive sentence summarization. arXiv preprint arXiv:1509.00685, 2015.

[100] Seonggi Ryang and Takeshi Abekawa. Framework of automatic text summarization using reinforcement learning. In Proceedings of the 2012 Joint Conference on Empirical Methods in Natural Language Processing and Computational Natural Language Learning, pages 256-265. Association for Computational Linguistics, 2012.

[101] Horacio Saggion, Juan-Manuel Torres-Moreno, Iria da Cunha, and Eric SanJuan. Multilingual summarization evaluation without human models. In Proceedings of the 23rd International Conference on Computational Linguistics: Posters, pages 1059-1067. Association for Computational Linguistics, 2010.

[102] K Sankar and L Sobha. An approach to text summarization. In Proceedings of the Third International Workshop on Cross Lingual Information Access: Addressing the Information Need of Multilingual Societies, pages 53-60. Association for Computational Linguistics, 2009.
[103] Kamal Sarkar, Mita Nasipuri, and Suranjan Ghose. Using machine learning for medical document summarization. International Journal of Database Theory and Application, 4(1):31-48, 2011.

[104] H Gregory Silber and Kathleen F McCoy. Efficiently computed lexical chains as an intermediate representation for automatic text summarization. Computational Linguistics, 28(4):487-496, 2002.

[105] Josef Steinberger and Karel Ježek. Evaluation measures for text summarization. Computing and Informatics, 28(2):251275, 2012.

[106] Ladda Suanmali, Naomie Salim, and Mohammed Salem Binwahlan. Fuzzy logic based method for improving text summarization. arXiv preprint arXiv:0906.4690, 2009.

[107] Hiroya Takamura and Manabu Okumura. Text summarization model based on the budgeted median problem. In Proceedings of the 18th ACM conference on Information and knowledge management, pages 1589-1592. ACM, 2009.

[108] Hideki Tanaka, Akinori Kinoshita, Takeshi Kobayakawa, Tadashi Kumano, and Naoto Kato. Syntax-driven sentence revision for broadcast news summarization. In Proceedings of the 2009 Workshop on Language Generation and Summarisation, pages 39-47. Association for Computational Linguistics, 2009.

[109] Doina Tatar, Andreea Diana Mihis, and Gabriela Serban. Top-down cohesion segmentation in summarization. In Proceedings of the 2008 Conference on Semantics in Text Processing, pages 389-397. Association for Computational Linguistics, 2008.

[110] Ha Nguyen Thi Thu and Dung Vu Thi Ngoc. Improve bayesian network to generating vietnamese sentence reduction. IERI Procedia, 10:190-195, 2014.

[111] Ivan Titov and Ryan T McDonald. A joint model of text and aspect ratings for sentiment summarization. In $A C L$, volume 8, pages 308-316. Citeseer, 2008.

[112] Oriol Vinyals and Quoc Le. A neural conversational model. arXiv preprint arXiv:1506.05869, 2015.

[113] Lu Wang and Wang Ling. Neural network-based abstract generation for opinions and arguments. arXiv preprint arXiv:1606.02785, 2016.

[114] Lu Wang, Hema Raghavan, Claire Cardie, and Vittorio Castelli. Query-focused opinion summarization for usergenerated content. arXiv preprint arXiv:1606.05702, 2016.

[115] Mark Wasson. Using leading text for news summaries: Evaluation results and implications for commercial summarization applications. In Proceedings of the 17th international conference on Computational linguistics-Volume 2, pages 1364-1368. Association for Computational Linguistics, 1998.

[116] Jen-Yuan Yeh, Hao-Ren Ke, Wei-Pang Yang, and I-Heng Meng. Text summarization using a trainable summarizer and latent semantic analysis. Information processing \& management, 41(1):75-95, 2005.

[117] Mehdi Yousfi-Monod and Violaine Prince. Sentence compression as a step in summarization or an alternative path in text shortening. In Coling'08: International conference on computational linguistics, pages 137-140, 2008.

[118] Lei Yu and Fuji Ren. A study on cross-language text summarization using supervised methods. In Natural Language 
Processing and Knowledge Engineering, 2009. NLP-KE 2009. International Conference on, pages 1-7. IEEE, 2009.

[119] Hongyuan Zha. Generic summarization and keyphrase extraction using mutual reinforcement principle and sentence clustering. In Proceedings of the 25th annual international ACM SIGIR conference on Research and development in information retrieval, pages 113-120. ACM, 2002.

[120] Jiaming Zhan, Han Tong Loh, and Ying Liu. Gather customer concerns from online product reviews-a text summarization approach. Expert Systems with Applications, 36(2):2107-2115, 2009.

[121] Renxian Zhang, Wenjie Li, Dehong Gao, and You Ouyang. Automatic twitter topic summarization with speech acts IEEE transactions on audio, speech, and language processing, 21(3):649-658, 2013.

[122] Markus Zopf, Eneldo Loza Mencia, and Johannes Fürnkranz. Beyond centrality and structural features: Learning information importance for text summarization. CoNLL 2016, page 84, 2016. 